\title{
Study of the method during Micro- EDM Process
}

\author{
Shuyang Liu, Zhihong Han \\ School of Mechanical \& Electronic Engineering, \\ Jingdezhen ceramic Institute, \\ Jiang Xi, China \\ hanliuyang@163.com
}

\begin{abstract}
In this paper, the work energy on the machined surface during electrical discharge machining (short in EDM) process were modeling analyzed based on the electron field emission theory, electron sputtering theory and the effect of resistance heat of electron current when the tool electrode is positive. A series of single-hole EDM experiments and the metallographic examinations were designed. The study shows that the concentration ratio of the work energy on the machined surface increase with the increase of the equivalent radius ratio of real discharging area and the area of workpiece end surface and the reduction of the interelectrode discharging current. While it can be obtained that the ratio of the resistance heat to the total work energy increase with the decrease of the equivalent radius ratio of real discharging area and the area of workpiece end surface and the increase of the interelectrode discharging current.
\end{abstract}

Keywords- EDM; field emission theory; Resistance heat; transforming heat; electrons sputtering energy

\section{INTRODUCTION}

As a kind of special machining technology, EDM has already been indispensable in processing ultra-hard materials and heat sensitive materials [1-3]. However, electrical discharge phenomena in EDM make both observation and theoretical analysis extremely difficult. Therefore, some questions still remain. First, the material removal mechanisms are not yet well understood. Second, what are the relationships between the energy distribution and working efficiency? Third, how the EDM process results in the energy distribution [4]? It shows that further research work is necessary for a better understanding of the nature of the phenomena involved in the EDM process.

Many scholars' research and related experiments have already shown that particles in the discharge channel were mainly on electric streams ejected from the cathode, and the effects of positive ions on the channel were very small [5]. Modern field-emission theories and thermal field emission theories, especially F-N equation descriptions, demonstrated that electrons escaping from surface needed to overcome surface barriers to work, which would translate into heat energy, and gave the reactive functions of material [6-8]. Mao, $\mathrm{C}$ et al. conducted that with longer pulse-on time, the power available between the anode and cathode becomes greater, thus strengthening discharge energy [9]. Liu et al. [10] derived electrodes complied with skin effect when flowing through the electrodes, which gave space boundary conditions for energy analysis of material removal [11].
In this paper, the energies on workpiece surface both in positive polarity EDM process were modeled and its related parameters were analyzed, based on electron field emission theory and the resistance heat reaction of electron flow when the tool electrode is positive. A set of machining blind-hole experiments with pole electrodes were carried out and investigated, and the related parameters that affect the working efficiencies were discussed. The experimental phenomena and results verify the rationality and effectiveness of the proposed model.

\section{THE SINGLE-HOLE EDM EXPERIMENTS WITH POLE ELECTRODES}

In order to analyses the mechanism and parameters of EDM processing, we designed single-hole EDM experiments with pole electrodes shown in Fig. 1, where $S w_{p}$ represents the pulse switch and $U_{s}$ is the voltage of discharging tube, $R_{p p}$ and $R_{n p}$ are the resistances of tool electrode and workpiece electrode respectively, $R_{p v}$ is the resistance of single discharging tube, and a number of pulse tubes is $n$.

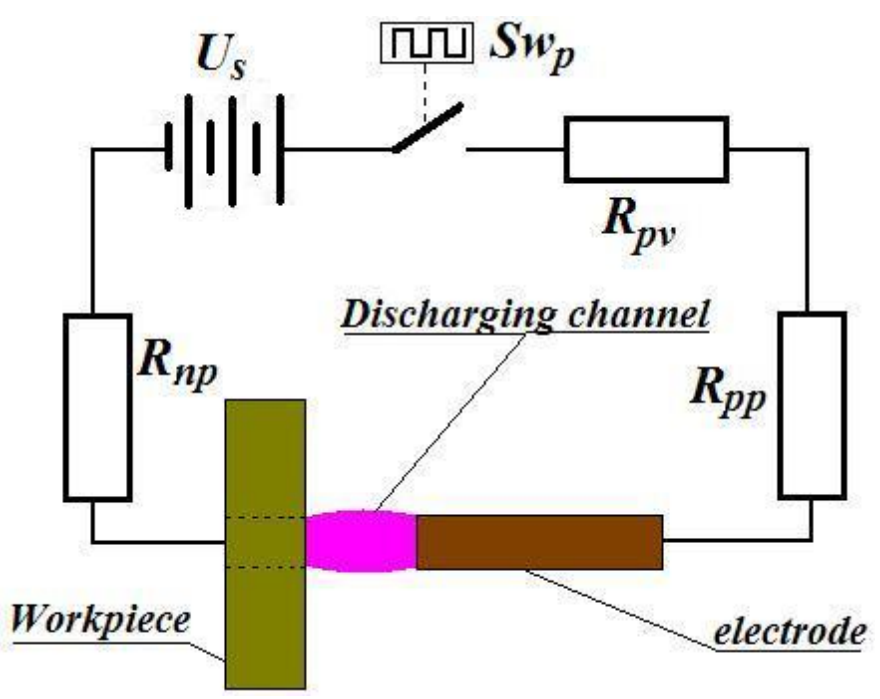

Fig. 1. Single-hole EDM experiments with pole electrode.

Related parameters of devices applied in the experiments are as follows:

Machine model: AGIE CHARMILLES SE2;;

Inputting power: $P_{i n}=10 \mathrm{kw}$;

Dielectric medium: kerosene 
The images of the bottom surface of EDMed blind hole by scanning electron microscope (SEM) are shown in Fig. 2, where the polarity of tool electrode is positive.
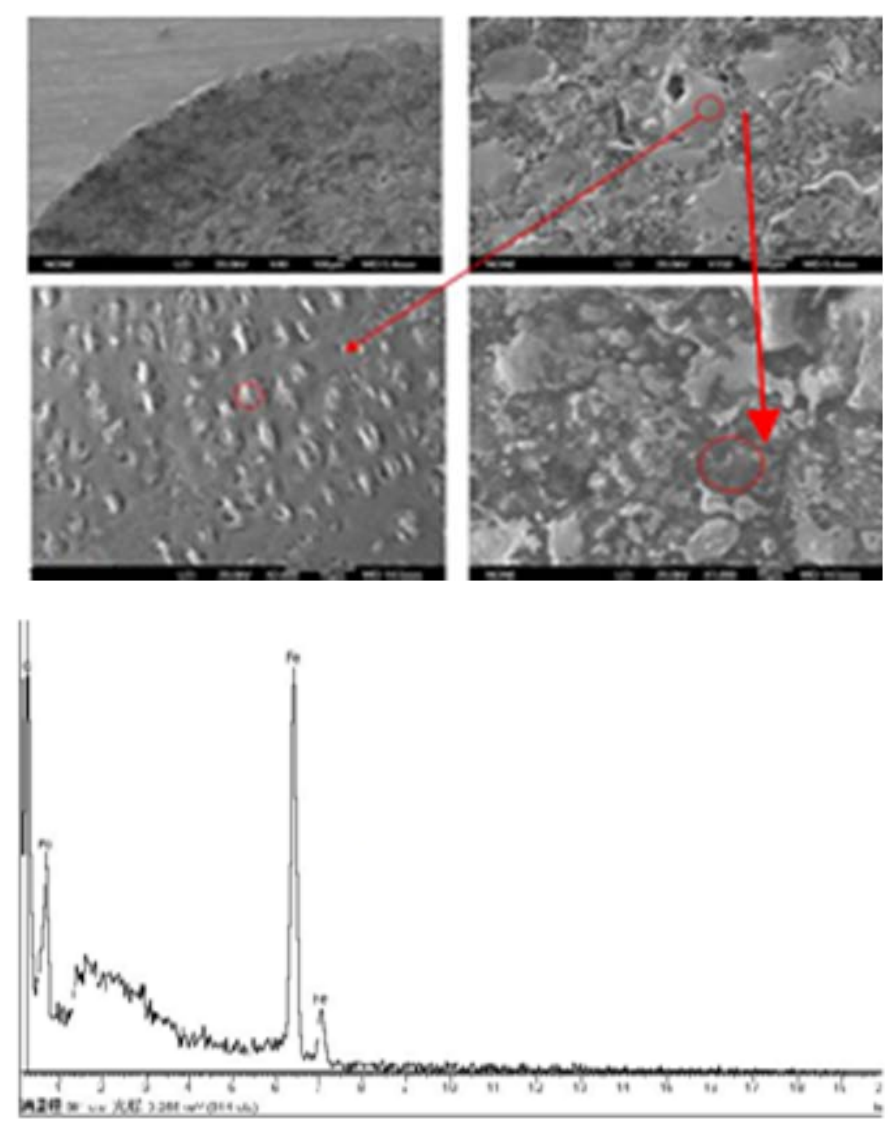

Fig. 2. SEM images of the bottom surface of EDMed blind hole..

It's clearly known by observing the microstructure of the machined surface and analyzing the corresponding metallographic pictures that:

(1) Fig. 2 shows that the electrode surface machined by positive polarity EDM process has obviously melted traces and the size distribution of melted spot is nonuniform, which states poorer thermal concentration degree of positive polarity EDM process during the single discharge cycle.

(2) From Fig. 2, it is speculated that the thermal corrosion of positive polarity machining is generated from inner substrate material to workpiece surface.

\section{THE WORK ENERGY ANALYSIS ON THE WORKPIECE \\ SURFACE OF POSITIVE EDM PROCESS}

According to the Skin Effect of electric charge, the electron current channel performs a shape as a concentric tube with two diameters $2 \pi\left(R_{2}-d_{a}\right) \times L$ and $\pi\left(R_{2}{ }^{2}-r^{2}\right) \times d_{a}$ respectively, shown in Fig. 3.

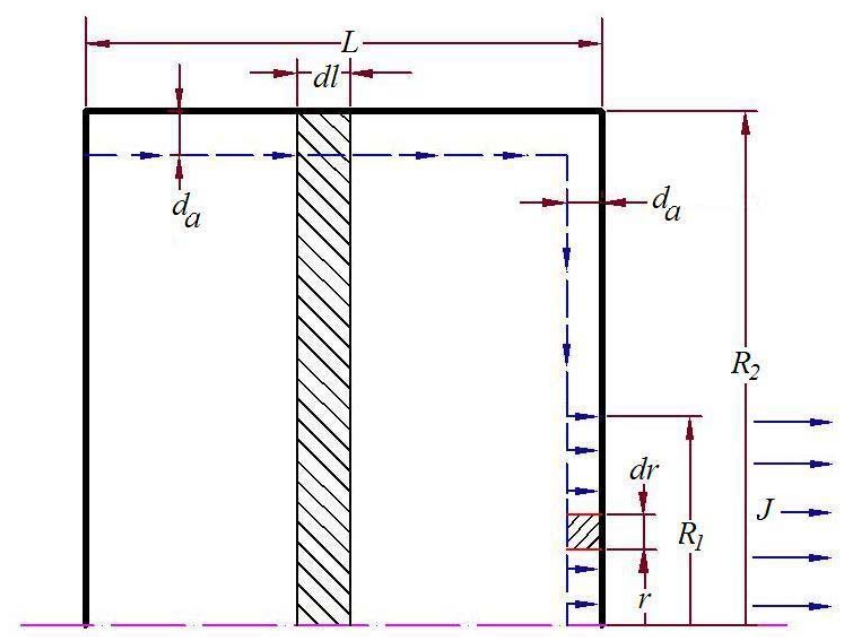

Fig. 3. The movement of electron current in workpiece electrode.

According to the Skin Effect of electric charge, the electron current channel performs a shape as a concentric tube with two diameters $2 \pi\left(R_{2}-d_{a}\right) \times L$ and $\pi\left(R_{2}{ }^{2}-r^{2}\right) \times d_{a}$ respectively, shown in Fig. 3.

Defining the ratio of the resistance heat to the total work energy on the area of $A_{1}$ as,

$$
\eta_{c}^{-}=\frac{Q_{s}^{\left(A_{1}^{-}\right)}}{Q^{\left(A_{1}^{-}\right)}}
$$

the ratio can be calculated as,

$$
\begin{aligned}
\eta_{c}^{-} & =\frac{\frac{2 \rho_{w}}{3 \pi \cdot d_{a}} \cdot\left(\frac{R_{2}}{R_{1}}-\frac{R_{1}{ }^{2}}{R_{2}{ }^{2}}+\frac{3}{4}\right) \cdot \int_{o}^{T_{o n}} J_{t}^{2} \cdot d t}{\frac{\pi R_{1}{ }^{2} \Phi}{e} \cdot \int_{o}^{T_{o n}} J_{t} \cdot d t+\frac{2 \rho_{w}}{3 \pi \cdot d_{a}} \cdot\left(\frac{R_{2}}{R_{1}}-\frac{R_{1}{ }^{2}}{R_{2}{ }^{2}}+\frac{3}{4}\right) \cdot \int_{o}^{T_{o n}} J_{t}^{2} \cdot d t} \\
& =\frac{C_{2} \cdot\left(4-4 \lambda^{3}+3 \lambda\right) \cdot \int_{o}^{T_{o n}} J_{t}^{2} \cdot d t}{C_{1} \cdot \lambda^{3} \cdot R_{2}{ }^{2} \cdot \int_{o}^{T_{o n}} J_{t} \cdot d t+C_{2} \cdot\left(4-4 \lambda^{3}+3 \lambda\right) \cdot \int_{o}^{T_{o n}} J_{t}^{2} \cdot d t}
\end{aligned}
$$

Taking the data of the single-hole EDM experiment of positive polarity listed as the example too, the varying tendency of $\eta_{c}^{-}$about $\lambda$ at different steady discharging current are shown in Fig. 4. Obviously, $\eta_{c}^{-}$is a decreasing function about $\lambda$ at certain $J_{t 3}$, and at some certain $\lambda, \eta_{c}^{-}$ gets higher with the increase of $J_{t 3}$. 


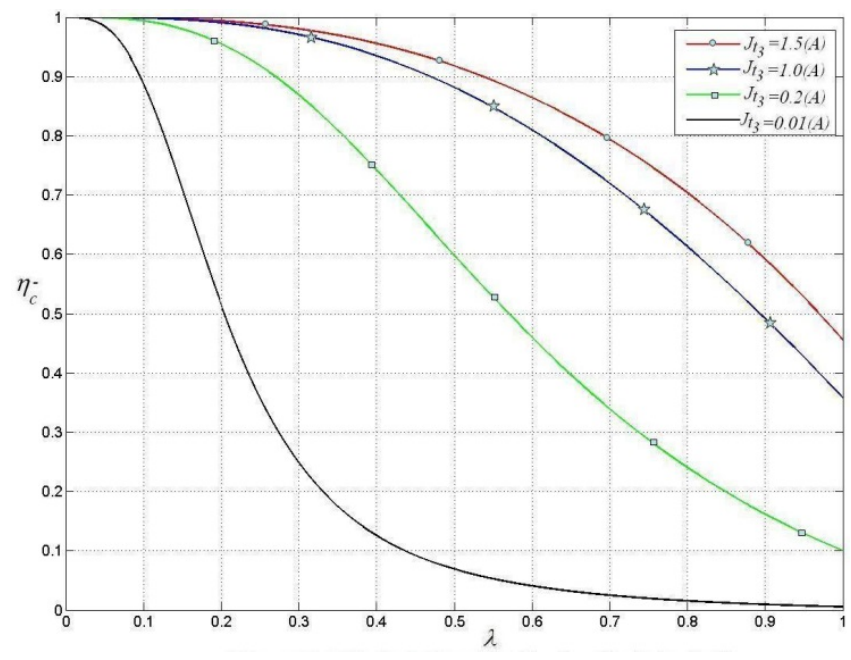

Fig. 4. The varying curves of $\eta_{\mathrm{c}}^{-}$about $\lambda$ at different $\mathrm{J}_{\mathrm{t} 3}$.

Fig. 5 shows the varying tendencies of $\eta_{c}^{-}$about $J_{t 3}$ at different equivalent radius ratios, obviously, $\eta_{c}^{-}$is an increasing function about $J_{t 3}$ at a certain $\lambda$, and $\eta_{Q}^{-}$gets smaller with the increase of $\lambda$ at some certain $J_{t 3}$,

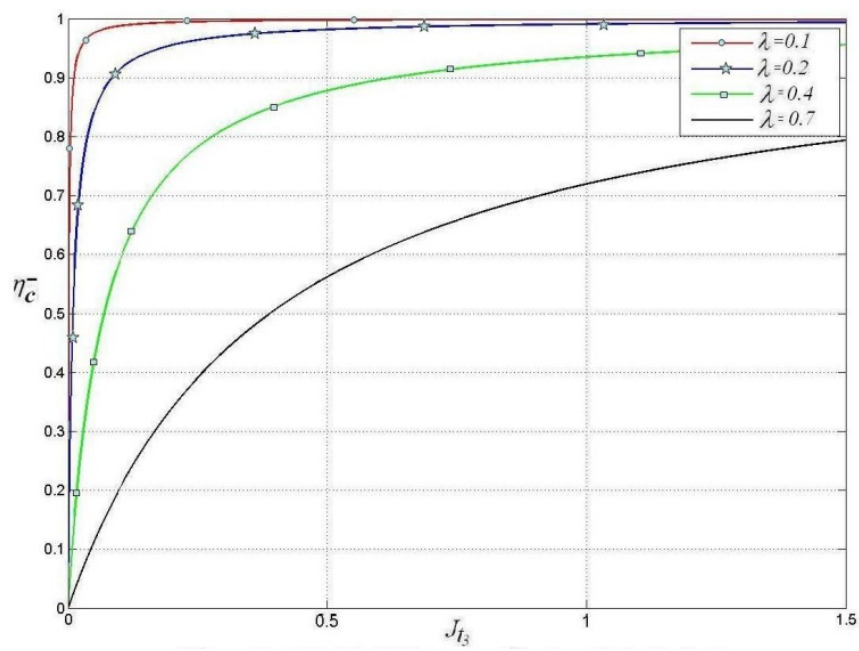

Fig. 5. The varying curves of $\eta_{\mathrm{c}}^{-}$about $\mathrm{J}_{\mathrm{t} 3}$ at different $\lambda$.

\section{CONCLUSIONS}

This study proposed the calculation equations about the work energy acting on the material removal during positive polarity EDM process and a series of single-hole EDM experiments and the metallographic examinations were designed to verify the model. The following conclusions are drawn from the experimental results:

(1) Based on the field emission theory and the effect of resistance heat, the work energy on the machined surface in positive polarity EDM process was studied, and the relevant equations were given. The related parameters that affect the working efficiencies were discussed.
(2) The calculation on the distribution of the work energies during the steady discharging stage of a pulse period shows that the concentration ratio of the work energy on the machined surface increase with the increase of the equivalent radius ratio of real discharging area and the area of workpiece end surface and the reduction of the interelectrode discharging current.

\section{REFERENCES}

[1] V. Yadav, V.K. Jain, and P.M. Dixit, "Thermal stresses due to electrical discharge machining”, Int. J. Mach. Tool. Manuf. vol. 42, pp. 877-888, 2002.

[2] R. Pérez, J. Carron, M. Rappaz, G. Wälder, B. Revaz, and R. Flükiger. Measurement and metallurgical modeling of the thermal impact of EDM discharg on steel, in Proceedings of the 15th International Symposium on Electromachining, ISEM XV, pp. 17-22, 2007.

[3] M.S. Murali, and S.H. Yeo. "Process simulation and residual stress estimations of micro-electrodischarge machining using finite element method”, Japanese J. Appl. Phys. vol. 44, no. 7A, pp. 5254-5263, 2005.

[4] B. Lauwers, J.P. Kruth, W. Liu, W. Eeraerts, B. Schacht, and P. Bleys, "Investigation of material removal mechanism in EDM of composite ceramic materials”, Mater. Process. Technol. vol. 149, pp. 347-352, 2004.

[5] S.Y. Liu,Y.M. Huang, and Y. Li. "A Plate capacitor model of the EDM process based on the field emission theory”, Int., J. Mach. Tool. Manuf. vol. 51, no. 7, pp. 653-659, 2004.

[6] R.H. Fowler, and L.W. Nordheim. "Electron emission in intense electric fields”, .Proc. Roy. Soc. Lund. Ser. A, vol. 119, pp. 173, 1928.

[7] E.L. Murphy, and R.H. Good. "Thermionic emission . field emission . and the transition region”, Phys. Rev. vol. 102, pp. 1464, 1956.

[8] R.H. Hare, R.M. Hill, and C.J. Budd. "Modeling charge injection and motion in solid dielectrics under high electric field", Plays. D: Appl. Phys. vol. 26, pp. 1084, 1993.

[9] Y.C. Lin, Y.F. Chen, C.T. Lin, and H.J. Tzeng, "Electrical discharge machining (EDM) characteristics associated with electrical discharge energy on machining of cemented tungsten carbide”, Mater. Manuf. Proc. vol. 23, no. 3-4, pp. 391-399, 2008.

[10] Y. Liu Yu, and F.L. Zhao, "Influences of skin-effect on micro-EDM”, J. Dalian Univ. Technol. no. 3, pp. 362-367, 2010. 